\title{
New Coordinate Vacuum Solution in Cosmological General
}

\section{Theory of Relativity}

\author{
Sangwha-Yi* \\ Department of Math, Taejon University 300-716, South Korea
}

*Corresponding Author: Sangwha-Yi, Department of Math, Taejon University 300-716, South Korea

Abstract: In the general relativity theory, we discover new vacuum solution by Einstein's gravity field equation. We investigate the new coordinate in cosmological general theory of relativity (CGTR).

Keywords: Cosmological General Theory of Relativity; Gravity Field Equation; New Coordinate Vacuum Solution

PACS Number: 04,04.90.+e,98.80,98.80.E

\section{INTRODUCTION}

We solve new vacuum solution by gravity field equation in cosmological general theory of relativity.

New spherical coordinate is

$d \tau^{2}=d t^{2}-\frac{1}{c^{2}}\left[d r^{2}+V(t, r)\left\{d \theta^{2}+\sin ^{2} \theta d \phi^{2}\right\}\right]$

$V(t, r)=C_{1}(a c t+b r)^{2}, \quad C_{1}=\frac{1}{b^{2}-a^{2}}$

$a, b, C_{1}$ is constant, $C$ is light's velocity.

In this time, Einstein's gravity equation is

$$
\begin{aligned}
& R_{t t}=\frac{\ddot{V}}{V}-\frac{\dot{V}^{2}}{2 V^{2}} \\
& =\frac{2 a^{2}}{(a c t+b r)^{2}}-\frac{1}{2} \frac{4 a^{2}}{(a c t+b r)^{2}}=0 \\
& R_{r r}=\frac{V^{\prime \prime}}{V}-\frac{1}{2} \frac{V^{2}}{V^{2}} \\
& =\frac{2 b^{2}}{(a c t+b r)^{2}}-\frac{1}{2} \frac{4 b^{2}}{(a c t+b r)^{2}}=0
\end{aligned}
$$

$R_{\theta \theta}=-\frac{\ddot{V}}{2}+\frac{V^{\prime \prime}}{2}-1$

$=-C_{1} a^{2}+C_{1} b^{2}-1=0$ 


$$
\begin{aligned}
& R_{\phi \phi}=\sin ^{2} \theta R_{\theta \theta}=0 \\
& R_{t r}=\frac{\dot{V}^{\prime}}{V}-\frac{\dot{V} V^{\prime}}{2 V^{2}} \\
& =\frac{2 C_{1} a b}{(a c t+b r)^{2}}-\frac{1}{2} \frac{4 C_{1} a b}{(a c t+b r)^{2}}=0
\end{aligned}
$$

In this time,

$$
\begin{aligned}
& V^{\prime}=2 C_{1} b(a c t+b r), \dot{V}=2 C_{1} a(a c t+b r), V^{\prime \prime}=2 C_{1} b^{2}, \ddot{V}=2 C_{1} a^{2} \\
& A^{\prime}=\frac{\partial A}{\partial r}, \dot{A}=\frac{1}{C} \frac{\partial A}{\partial t}
\end{aligned}
$$

\section{New Vacuum Solution in Cosmological General Theory of Relativity}

Hence, new vacuum solution is

$$
d \tau^{2}=d t^{2}-\frac{1}{c^{2}}\left[d r^{2}+\frac{1}{b^{2}-a^{2}}(a c t+b r)^{2}\left\{d \theta^{2}+\sin ^{2} \theta d \phi^{2}\right\}\right]
$$

$a, b, C_{1}$ are constant, $C$ is light's velocity.

In this time, if $r^{\prime}$ is

$$
r^{\prime}=\frac{1}{\sqrt{b^{2}-a^{2}}}(a c t+b r)
$$

As

$$
d r^{\prime}=\frac{1}{\sqrt{b^{2}-a^{2}}}(a c d t+b d r)
$$

Or

$$
d r=\frac{\sqrt{b^{2}-a^{2}}}{b} d r^{\prime}-\frac{a}{b} c d t
$$

If new solution $\mathrm{Eq}(7)$ is inserted by transformation $\mathrm{Eq}(8)$,

$d r^{2}=\frac{b^{2}-a^{2}}{b^{2}} d r^{2}-2 \frac{a}{b^{2}} \sqrt{b^{2}-a^{2}} d r^{\prime} c d t+\frac{a^{2}}{b^{2}} c^{2} d t^{2}$

In this time, if $\alpha_{0}$ is

$$
\alpha_{0}=\frac{a}{b}
$$

Hence, proper time $d \tau$ of new solution is

$$
d \tau^{2}=\left(1-\alpha_{0}^{2}\right) d t^{2}+2 \alpha_{0} \sqrt{1-\alpha_{0}^{2}} d r^{\prime} \frac{d t}{c}-\frac{1}{c^{2}}\left[\left(1-\alpha_{0}{ }^{2}\right) d r^{2}+r^{2}\left\{d \theta^{2}+\sin ^{2} \theta d \phi^{2}\right\}\right]
$$

In this time, if $d t^{\prime}$ is

$d t^{\prime}=\sqrt{1-\alpha_{0}^{2}} d t$

Therefore, new solution is 
$d \tau^{2}=d t^{2}+2 \alpha_{0} d r^{\prime} \frac{d t^{\prime}}{c}-\frac{1}{c^{2}}\left[\left(1-\alpha_{0}^{2}\right) d r^{2}+r^{2}\left\{d \theta^{2}+\sin ^{2} \theta d \phi^{2}\right\}\right]$

If we rewrite $d t, d r$ instead of $d t^{\prime}, d r^{\prime}$, the proper time $d \tau$ of new solution is

$d \tau^{2}=d t^{2}+2 \alpha_{0} d r \frac{d t}{c}-\frac{1}{c^{2}}\left[\left(1-\alpha_{0}^{2}\right) d r^{2}+r^{2}\left\{d \theta^{2}+\sin ^{2} \theta d \phi^{2}\right\}\right]$

Therefore, new spherical solution in general relativity theory is

$d \tau^{2}=d t^{2}+2 \alpha_{0} d r \frac{d t}{c}-\frac{1}{c^{2}}\left[\left(1-\alpha_{0}^{2}\right) d r^{2}+r^{2}\left\{d \theta^{2}+\sin ^{2} \theta d \phi^{2}\right\}\right]$

$\alpha_{0} \neq 1, \quad \alpha_{0}$ is constant

In this time, the coordinate transformation in cosmological general theory of relativity [1-3] is

$r \rightarrow I \Omega\left(t_{0}\right), t \rightarrow t \quad$,

$t_{0}$ is cosmological time. $\Omega\left(t_{0}\right)$ is the ratio of universe's expansion in cosmological time $t_{0}$

Hence, this vacuum solution is by the coordinate transformation in cosmological general theory of relativity,

$$
\begin{aligned}
& d \tau^{2}=d t^{2}+2 \alpha_{0} \Omega\left(t_{0}\right) d r \frac{d t}{C}-\frac{\Omega^{2}\left(t_{0}\right)}{c^{2}}\left[\left(1-\alpha_{0}{ }^{2}\right) d r^{2}+r^{2}\left\{d \theta^{2}+\sin ^{2} \theta d \varphi^{2}\right\}\right] \\
& \alpha_{0} \neq 1, \quad \alpha_{0} \text { is constant }
\end{aligned}
$$

\section{CONCLUSION}

In the general relativity theory, we discover new vacuum solution by Einstein's gravity field equation. We investigate the new coordinate in cosmological general theory of relativity.

\section{REFERENCES}

[1]S.Yi, "Cosmological General Theory of Relativity", International Journal of Advanced Research in Physical Science,8,2,(2021),pp 22-26

[2]S.Yi, "PMBH Theory of Representation of Gravity Field Equation and Solutions, Hawking Radiation in Data General Relativity Theory”, International Journal of Advanced Research in Physical Science,5,9,(2018),pp 36-45

[3]S.Yi, "Yukawa Potential in Klein-Gordon Equation in Cosmological Special Theory of Relativity", International Journal of Advanced Research in Physical Science,8,3,(2021),pp 16-18

[4]S.Weinberg,Gravitation and Cosmology(John wiley \& Sons,Inc,1972)

[5]P.Bergman,Introduction to the Theory of Relativity(Dover Pub. Co.,Inc., New York,1976), Chapter V

[6]C.Misner, K,Thorne and J. Wheeler, Gravitation(W.H.Freedman \& Co.,1973)

[7]S.Hawking and G. Ellis,The Large Scale Structure of Space-Time(Cam-bridge University Press,1973)

[8]R.Adler,M.Bazin and M.Schiffer,Introduction to General Relativity(McGraw-Hill,Inc.,1965)

[9]E.Kasner, Am. J. Math. 43, 217(1921)

[10]G.Birkoff,Relativity and Modern Physics(Harvard University Press,1923),p.253 
[11]T.Kaluza, Berl. Ber. 996(1921); O. Klein, Z. Phys. 37, 895(1926)

[12]Y. Cho, J. Math. Phys. 16, 2029(1975); Y. Cho and P. Freund, Phys. Rev. D12, 1711(1975)

[13]P. van Nieuwenhuizen, Phys. Rep. 68. 189(1981)

Citation: Sangwha-Yi (2021). New Coordinate Vacuum Solution in Cosmological General Theory of Relativity. International Journal of Advanced Research in Physical Science (IJARPS) 8(4), pp.26-29, 2021.

Copyright: (C) 2021 Authors, This is an open-access article distributed under the terms of the Creative Commons Attribution License, which permits unrestricted use, distribution, and reproduction in any medium, provided the original author and source are credited. 